\title{
On the Ground State to Hamiltonian Elliptic System with Choquard's Nonlinear Term
}

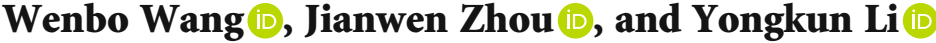 \\ School of Mathematics and Statistics, Yunnan University, Kunming, 650500 Yunnan, China \\ Correspondence should be addressed to Jianwen Zhou; jwzhou@ynu.edu.cn
}

Received 14 January 2020; Accepted 23 March 2020; Published 5 May 2020

Academic Editor: Wen-Xiu Ma

Copyright ( 2020 Wenbo Wang et al. This is an open access article distributed under the Creative Commons Attribution License, which permits unrestricted use, distribution, and reproduction in any medium, provided the original work is properly cited.

In the present paper, we consider the following Hamiltonian elliptic system with Choquard's nonlinear term $\left\{\begin{array}{l}-\Delta u+V(x) u=\int_{\Omega}\left(G(v(y)) /|x-y|^{\beta}\right) d y g(v) \text { in } \Omega, \\ -\Delta v+V(x) v=\int_{\Omega}\left(F(u(y)) /|x-y|^{\alpha}\right) d y f(u) \text { in } \Omega, \text { where } \Omega \subset \mathbb{R}^{N} \text { is a bounded domain with a smooth boundary, } 0<\alpha<N, 0< \\ u=0, v=0 \text { on } \partial \Omega,\end{array}\right.$ $\beta<N$, and $F$ is the primitive of $f$, similarly for $G$. By establishing a strongly indefinite variational setting, we prove that the above problem has a ground state solution.

\section{Introduction and Main Results}

In this paper, we deal with the existence of ground state solutions for the following Hamiltonian elliptic system with Choquard's nonlinear term:

$$
\left\{\begin{array}{l}
-\Delta u+V(x) u=\int_{\Omega} \frac{G(v(y))}{|x-y|^{\beta}} d y g(v) \text { in } \Omega, \\
-\Delta v+V(x) v=\int_{\Omega} \frac{F(u(y))}{|x-y|^{\alpha}} d y f(u) \text { in } \Omega, \\
u=0, v=0 \text { on } \partial \Omega,
\end{array}\right.
$$

where $\Omega \subset \mathbb{R}^{N}$ is a bounded domain with a smooth boundary, $N \geq 3,0<\alpha<N, 0<\beta<N$, and $F$ is the primitive of $f$, similarly for $G$. For a single equation in whole space, $\mathbb{R}^{N}$ is closely related to the Choquard-Pekar equation:

$-\Delta u+V(x) u=\int_{\mathbb{R}^{N}} \frac{F(u(y))}{|x-y|^{\mu}} d y f(u)$, in $\mathbb{R}^{N}, \quad 0<\mu<N$.
When $N=3, \mu=1, V(x) \equiv 1$, and $f(u)=u$, equation (2) has appeared in several contexts of quantum physics. In 1954, Pekar used equation (2) to describe a polaron at rest in quantum theory. In 1976, to model an electron trapped in its own hole, P. Choquard considered (2) as a certain approximation to Hartree-Fock's theory of one component plasma (see $[1,2])$. In some particular cases, $(2)$ is also known as the Schrödinger-Newton equation which was introduced by Penrose in [3] to describe the self-gravitational collapse of a quantum mechanical wave function.

For $N=3, \mu=1, V(x) 1$, and $f(u)=u$, the existence of ground states of (2) was obtained in [4] by variational methods. Later, Moroz and Van Schaftingen [2] investigated the regularity, radial symmetry, and asymptotic behavior at infinity of positive solutions for a generalized Choquard equation. Other results involving existence, multiplicity, and concentration of Choquard's problems can be found in [5-12] and the references therein.

We also observed that Bhattarai [13] considered the coupled Choquard-type fractional Schrodinger systems: 


$$
\left\{\begin{array}{l}
(-\Delta)^{\alpha} u+\omega_{1} u=\lambda_{1} \int_{\mathbb{R}^{N}} \frac{|u(y)|^{p_{1}}}{|x-y|^{\beta}} d y|u|^{p_{1}-2} u+\int_{\mathbb{R}^{N}} \frac{|v(y)|^{q}}{|x-y|^{\beta}} d y|u|^{q-2} u, \text { in } \mathbb{R}^{N}, \\
(-\Delta)^{\alpha} v+\omega_{2} v=\lambda_{2} \int_{\mathbb{R}^{N}} \frac{|v(y)|^{p_{2}}}{|x-y|^{\beta}} d y|v|^{p_{2}-2} v+\int_{\mathbb{R}^{N}} \frac{|u(y)|^{q}}{|x-y|^{\beta}} d y|v|^{q-2} v, \text { in } \mathbb{R}^{N},
\end{array}\right.
$$

where $\omega_{1}$ and $\omega_{2}$ appear as the Lagrange multipliers, $N \geq 2$, $0<\alpha<1, \quad 0<\beta<N, \quad 2 \leq p_{1}, \quad p_{2}$, and $q<(N+2 \alpha+\beta) / N$. Bhattarai looked for minimizers of the $L^{2}$-constrained minimization problem via concentration compactness techniques. Recently, Giacomoni et al. [14] were also concerned with the coupled Hardy-Littlewood-Sobolev critical nonlinearity fractional Schrödinger system in a smooth bounded domain $\Omega \subset \mathbb{R}^{N}$ :

$$
\left\{\begin{array}{l}
(-\Delta)^{\theta} u=\lambda|u|^{q-2} u+\left(\int_{\Omega} \frac{|v(y)|^{2_{\mu}^{*}}}{|x-y|^{\mu}} d y\right)|u|^{2_{\mu}^{*}-2} u \text { in } \Omega, \\
(-\Delta)^{\theta} v=\delta|v|^{q-2} v+\left(\int_{\Omega} \frac{|u(y)|^{2_{\mu}^{*}}}{|x-y|^{\mu}} d y\right)|v|^{2_{\mu}^{*}-2} v \operatorname{in} \Omega,
\end{array}\right.
$$

where $2_{\mu}^{*}=(2 N-\mu) /(N-2 \theta), 0<q<2$. By minimizing over a suitable subset of Nehari's manifold, they proved the existence of at least two nontrivial solutions for a suitable range of $\lambda$ and $\delta$. For problems involving Hardy-LittlewoodSobolev's critical exponent problems, please see [15-17] and the references therein. Among them, a strongly indefinite Choquard equation was studied in [17].

Motivated by the papers mentioned above, in particular, the papers $[13,14,17]$, the purpose of the present paper is to investigate the ground state solution of problem (1). To the best of our knowledge, there is no work concerning the existence of ground state solutions to the Choquard-type Hamiltonian elliptic system. For the Hamiltonian elliptic system, we refer the readers to the papers [18-22] and the references therein.

Throughout this paper, we will always assume $N \geq 3$ and we suppose that $V, g$, and $f$ satisfy the following assumptions.

(V) $V(x) \in L^{\infty}(\Omega, \mathbb{R}), 0$ lies in a gap of $\sigma(-\Delta+V(x))$, where $\sigma(-\Delta+V(x))$ is the spectrum of the operator $-\Delta+V(x)$.

$\left(\mathrm{H}_{1}\right) f, g \in C(\mathbb{R}, \mathbb{R})$.

$\left(\mathrm{H}_{2}\right) f(u)=o(u)$ as $u \longrightarrow 0, g(v)=o(v)$ as $v \longrightarrow 0$.

$\left(\mathrm{H}_{3}\right) \limsup _{u \rightarrow \infty}\left(f(u) / u^{p}\right)<\infty, \limsup _{v \rightarrow \infty}\left(g(v) / v^{q}\right)<\infty$, where $p, q>1$ satisfy

$$
\frac{N-(\alpha / 2)}{(p+1) N}+\frac{N-(\beta / 2)}{(q+1) N}>\frac{N-2}{N} .
$$

$\left(\mathrm{H}_{4}\right)$ There exists $\theta>2$, such that $u, v \in \mathbb{R}$ :

$$
\begin{aligned}
& 0 \leq \theta F(u) \leq f(u) u, \\
& 0 \leq \theta G(v) \leq g(v) v .
\end{aligned}
$$

$\left(\mathrm{H}_{5}\right) F(u)>0$ for $u \neq 0$ and $G(v)>0$ for $v \neq 0$.

Before stating our main result, we review the definition of $(s, t)$ ground state solution about (1). We call $(u, v) \neq$ $(0,0)$ as a $(s, t)$ ground state solution of (1) in work space $E$ (see Section 2); if $(\tilde{u}, \tilde{v})$ is another $(s, t)$ weak solution (see Definition 2), then the corresponding energy functional $I(u, v) \leq I(\tilde{u}, \tilde{v})$, where $I$ will be defined in equation (27) or equation (32).

Theorem 1. Suppose that $H_{1}, H_{2}, H_{3}, H_{4}$ and $H_{5}$ are satisfied. Then (1) has a $(s, t)$ ground state solution.

To prove Theorem 1, here we use a minimizing argument based on the ideas developed in [23]. We are concerned with system (1) in whole space $\mathbb{R}^{N}$ involving the Hamiltonian elliptic system with Choquard's nonlinear term; it is a nonlocal problem, which brings about two obstacles. One is to check the linking structure, and the other one is to prove the boundedness of the corresponding (PS) sequences. To avoid these obstacles, we shall deal with our problem in bounded domain. Indeed, the difficulty is still there for the problem on $\mathbb{R}^{N}$. It is worth pointing out that the monotonicity condition like [23] is not required on the nonlinear terms $f$ and $g$; it prevents us from using the standard way (see, e.g., $[23,24])$ to check that the minimizer is a critical point. Via the basic leitmotiv from Proposition 3.2 of [25], we shall use the deformation lemma to prove it (see Lemma 13.. We end this section by giving our arrangements of this paper. In Section 2, we establish the variational settings about (1). In Section 3, we provide some lemmas and then prove Theorem 1 .

\section{Variational Settings}

For the system

$$
\left\{\begin{array}{l}
-\Delta u+V(x) u=\int_{\Omega} \frac{G(v(y))}{|x-y|^{\beta}} d y g(v), \text { in } \Omega, \\
-\Delta v+V(x) v=\int_{\Omega} \frac{F(u(y))}{|x-y|^{\alpha}} d y f(u), \text { in } \Omega,
\end{array}\right.
$$


if $F(u)=|u|^{\theta+1}$, by Hardy-Littlewood-Sobolev's inequality (see Theorem 4.3 in [26]), then the term

$$
\int_{\Omega} \int_{\Omega} \frac{F(u(y)) F(u(x))}{|x-y|^{\alpha}} d y d x,
$$

is well defined if $F(u) \in L^{r}(\Omega)$ for $t>1$ such that $(2 / r)+(\alpha /$ $N)=2$. In view of Sobolev's embedding theorem, it requires that $1 \leq(\theta+1) r \leq 2 N /(N-2)$, which leads us to assume that

$$
1-\frac{\alpha}{2 N} \leq \theta-1 \leq \frac{2 N-\alpha}{N-2}
$$

Since we restrict $p \geq 1, q \geq 1$ and

$$
\frac{N-(\alpha / 2)}{(p+1) N}+\frac{N-(\beta / 2)}{(q+1) N}>\frac{N-2}{N} .
$$

So it holds that $1-(\alpha / 2 N) \leq p+1$ and $1-(\beta / 2 N) \leq q+1$ but $p$ or $q$ could be supercritical in the sense that

$$
\begin{aligned}
p+1 & >\frac{2 N-\alpha}{N-2} \\
\text { or } q+1> & \frac{2 N-\beta}{N-2} .
\end{aligned}
$$

We remark that $p \geq 1$ is used in (38) and similarly for $q \geq 1$. Since $p$ or $q$ could be supercritical, we need the fractional Sobolev spaces (see, e.g., $[19,22,27])$. According to $\mathrm{H}_{4}$, we can choose $s, t>0$ with $s+t=2$, such that

$$
\begin{aligned}
& p+1<\frac{N}{N-2 s}\left(2-\frac{\alpha}{N}\right) \\
& q+1<\frac{N}{N-2 t}\left(2-\frac{\beta}{N}\right) .
\end{aligned}
$$

Denote $S=-\Delta+V(x)$. Since the effective domain $\mathscr{D}(S)=\mathscr{D}\left(S^{*}\right)$ and $S$ is symmetric, so $S$ is self-adjoined on $L^{2}(\Omega)$. Since the self-adjoint operator is closed, according to the polar decomposition theorem (Theorem VIII.32 in [28] and jointly with Theorem 3.2 and 3.3, Ch IV in [29]), it holds that there is a positive self-adjoint operator $|S|$ (in fact $\left.|S|=\sqrt{S^{*} S}\right)$, with $\mathscr{D}(|S|)=\mathscr{D}(S)$ and a partial isometry $U$ such that $S=U|S|,|S|$ and $U$ are uniquely determined and

$$
\begin{gathered}
S U=U S, \\
|S| U=U|S| .
\end{gathered}
$$

It is well known that $U$ and $|S|$ are both self-adjoint operators on $L^{2}(\Omega)$. In view of Theorem 3.35 in Chapter $\mathrm{V}$ of [30] and Corollary 5.5.6 in [31], there is a unique square root operator $Q$ such that $Q^{2}=|S|$, furthermore, $Q U=U Q$. Denote $|S|^{1 / 2}:=Q$. Let

$$
\begin{aligned}
& A^{s}:=|S|^{s / 2}, \\
& A^{t}:=|S|^{t / 2} .
\end{aligned}
$$

According to $[19,27]$, we consider a basis of $L^{2}(\Omega)$ constituted by eigenfunctions $\left\{\phi_{n}\right\}$ of

$$
-\Delta u+V(x) u=\lambda u \text { in } \Omega, \quad u=0 \text { on } \partial \Omega,
$$

with associated eigenvalues $\lambda_{n}$. If for $u \in L^{2}(\Omega)$, we write $u=\sum_{n=1}^{\infty} a_{n} \phi_{n}$, then the effective domain of $A^{s}$ and $A^{t}$ are

$$
\begin{aligned}
& E^{s}:=\mathscr{D}\left(A^{s}\right)=\left\{u \in L^{2}(\Omega): \sum_{n=1}^{\infty} \lambda_{n}^{s}\left|a_{n}\right|^{2}<\infty\right\}, \\
& E^{t}:=\mathscr{D}\left(A^{t}\right)=\left\{u \in L^{2}(\Omega): \sum_{n=1}^{\infty} \lambda_{n}^{t}\left|a_{n}\right|^{2}<\infty\right\} .
\end{aligned}
$$

They are two Hilbert spaces endowed with the following inner product, respectively,

$$
\begin{aligned}
\langle u, v\rangle_{s} & =\left\langle A^{s} u, A^{s} v\right\rangle_{L^{2}}, \\
\langle\varphi, \psi\rangle_{t} & =\left\langle A^{t} \varphi, A^{t} \psi\right\rangle_{L^{2}},
\end{aligned}
$$

where

$$
\begin{aligned}
A^{s}: E^{s} & \longrightarrow L^{2}(\Omega), \\
u & \mapsto \sum_{n=1}^{\infty} \lambda_{n}^{s / 2} a_{n} \phi_{n},
\end{aligned}
$$

is an isometric isomorphism. So, $A^{s}$ has the inverse $\left(A^{s}\right)^{-1}$, and we denote $A^{-s}=\left(A^{s}\right)^{-1}$, similarly for $A^{t}$. Set $E=E^{s} \times$ $E^{t}$, then $E$ is a Hilbert space with the inner product and norm

$$
\begin{aligned}
\langle z, \eta\rangle & =\langle u, \varphi\rangle_{s}+\langle v, \psi\rangle_{t} \\
\|z\|^{2} & =\|u\|_{s}+\|v\|_{t}
\end{aligned}
$$

for $z=(u, v), \eta=(\varphi, \psi) \in E$. We recall the embedding theorem (see [32]); for $r>0$, the embedding $E^{r} \longrightarrow L^{k}\left(\mathbb{R}^{N}\right)$ is continuous for $1 \leq k \leq(2 N /(N-2 r))$ and is compact for $1 \leq k<(2 N /(N-2 r))$. We also consider the bounded selfadjoint operator $L: E \longrightarrow E$ defined as follows, for $z=(u, v)$, $\eta=(\varphi, \psi)$ :

$$
\langle L z, \eta\rangle=\left\langle A^{s} U u, A^{t} \psi\right\rangle_{L^{2}}+\left\langle A^{t} v, A^{s} U \varphi\right\rangle_{L^{2}}
$$

A natural question will be asked whether the operator $L: E \longrightarrow E$ is well defined. Here, we only check if $\left\langle A^{s} U u\right.$, $\left.A^{t} \psi\right\rangle_{L^{2}}$ is well defined. In fact, noting that $A^{s}$ and $U$ are self-adjoint operators, we infer that

$$
\left\langle A^{s} U u, A^{t} \psi\right\rangle_{L^{2}}=\left\langle U u, A^{2} \psi\right\rangle_{L^{2}}=\left\langle u, U|S| \psi_{L^{2}}=\langle u, S \psi\rangle_{L^{2}} .\right.
$$


By the complex interpolation theory (see Chapter 1.15 of [32]), we get

$$
E^{s}=\left[L^{2}(\Omega), H^{2}(\Omega)\right]_{1-(s / 2)} .
$$

So $\left\langle A^{s} U u, A^{t} \psi\right\rangle_{L^{2}}<\infty$.

Next, we have $L$ that has only two eigenvalues -1 and 1 , whose corresponding eigenspaces are

$$
\begin{aligned}
& E^{+}=\left\{\left(u, A^{-t} A^{s} U u\right): u \in E^{s}\right\}, \\
& E^{-}=\left\{\left(u,-A^{-t} A^{s} U u\right): u \in E^{s}\right\}
\end{aligned}
$$

Clearly, $E=E^{+} \oplus E^{-}$. Indeed, 0 lies in a gap of $\sigma((?+$ $V(x))$ ), and using Theorem 3.3 in Chapter IV of [29], we get

$$
L^{2}(\Omega)=L^{+} \oplus L^{-}
$$

where $L^{ \pm}=u \in L^{2}(\Omega): U u= \pm u$.
Similar to Proposition 1.1 in [19], for $z=(u, v)$, we can easily get

$$
L z=\left(A^{-s} A^{t} U v, A^{-t} A^{s} U u\right) .
$$

We consider the eigenvalue problem $L z=\lambda z$ in $E$, which yields that

$$
\lambda^{2} v=A^{-t} A^{s} U A^{-s} A^{t} U v=U U v=U\left(v^{+}+v^{-}\right)=v .
$$

Here, we have used the fact that $A^{s} U=U A^{s}$ and $A^{t} U=$ $U A^{t}$ (see [22]).

We consider the corresponding energy functional for (7):

$$
\begin{aligned}
I(u, v)= & \int_{\Omega} A^{s} U u A^{t} v d x-\frac{1}{2} \int_{\Omega} \int_{\Omega} \frac{F(u(y)) F(u(x))}{|x-y|^{\alpha}} d y d x \\
& -\frac{1}{2} \int_{\Omega} \int_{\Omega} \frac{G(v(y)) G(v(x))}{|x-y|^{\beta}} d y d x, \quad(u, v) \in E .
\end{aligned}
$$

Similarly ([27], p. 61), under our assumptions, using the fact that $A^{s} U$ and $A^{t}$ are linear, jointly with Lebesgue's dominated convergence theorem, the Gateaux derivative of $I$ in the direction $\eta=(\varphi, \psi)$ at $z=(u, v)$ is defined as

$$
\begin{aligned}
D I(z, \eta)= & \lim _{l \rightarrow 0} \frac{I(z+l \eta)-I(z)}{l}=\left\langle A^{s} U u, A^{t} \psi\right\rangle_{L^{2}}+\left\langle A^{s} U \phi, A^{t} v\right\rangle_{L^{2}} \\
& -\lim _{l \rightarrow 0} \frac{1}{2 l} \int_{\Omega} \int_{\Omega} \frac{F[u(y)+l \varphi(y)] F[u(x)+l \varphi(y)]-F[u(y)] F[u(x)]}{|x-y|^{\alpha}} d y d x \\
& -\lim _{l \rightarrow 0} \frac{1}{2 l} \int_{\Omega} \int_{\Omega} \frac{G[v(y)+l \psi(y)] G[v(x)+l \psi(y)]-G[v(y)] G[v(x)]}{|x-y|^{\beta}} d y d x \\
= & \left\langle A^{s} U u, A^{t} \psi\right\rangle_{L^{2}}+\left\langle A^{s} U \phi, A^{t} v\right\rangle_{L^{2}}-\int_{\Omega} \int_{\Omega} \frac{F(u(y)) f(u(x)) \varphi(x)}{|x-y|^{\alpha}} d y d x \\
& -\int_{\Omega} \int_{\Omega} \frac{G(v(y)) g(v(x)) \psi(x)}{|x-y|^{\alpha}} d y d x .
\end{aligned}
$$

Clearly, $D I(z, \eta)$ is linear bounded about $\eta$ and continuous about $z$. Thus, $I \in C^{1}(E, \mathbb{R})$. And its Fréchet derivative is given by

$$
\begin{aligned}
\left\langle I^{\prime}(z), \eta\right\rangle= & \left\langle A^{s} U u, A^{t} \psi\right\rangle_{L^{2}}+\left\langle A^{s} U \phi, A^{t} v\right\rangle_{L^{2}} \\
& -\int_{\Omega} \int_{\Omega} \frac{F(u(y)) f(u(x)) \varphi(x)}{|x-y|^{\alpha}} d y d x \\
& -\int_{\Omega} \int_{\Omega} \frac{G(v(y)) g(v(x)) \psi(x)}{|x-y|^{\alpha}} d y d x
\end{aligned}
$$

Definition 2. We say that $z=(u, v) \in E \backslash\{0\}$ is a $(s, t)$ weak solution of (7), if for each $\eta=(\varphi, \psi) \in E$, there holds

$$
\begin{aligned}
& \left\langle A^{s} U u, A^{s} \psi\right\rangle_{L^{2}}+A^{s} U \varphi, A^{t} v_{L^{2}} \\
& =\int_{\Omega} \int_{\Omega} \frac{F(u(y)) f(u(x)) \varphi(x)}{|x-y|^{\alpha}} d y d x \\
& \quad-\int_{\Omega} \int_{\Omega} \frac{G(v(y)) g(v(x)) \psi(x)}{|x-y|^{\beta}} d y d x .
\end{aligned}
$$


It is easy to check that the critical point $(u, v)$ of $I$ is a $(s, t)$ weak solution of (7). Moreover, for $z=(u, v) \in E, z=$ $z^{+}+z^{-}$, in view of Lemma 2.1 in [19], it holds that

$$
z^{ \pm}=\left(\frac{u \pm A^{-s} A^{t} U v}{2}, \frac{v \pm A^{-t} A^{s} U u}{2}\right)
$$

Therefore,

$$
\begin{aligned}
I(z)= & \frac{1}{2}\left(\left\|z^{+}\right\|^{2}-\left\|z^{-}\right\|^{2}\right)-\frac{1}{2} \int_{\Omega} \int_{\Omega} \frac{F(u(y)) F(u(x))}{|x-y|^{\alpha}} d y d x \\
& -\frac{1}{2} \int_{\Omega} \int_{\Omega} \frac{G(v(y)) G(v(x))}{|x-y|^{\beta}} d y d x .
\end{aligned}
$$

Remark 3. If $\inf _{x \in \Omega} V(x)>0$, then $U=I$.

\section{Existence of Ground States}

Following [23] (in page 3804), we introduce the generalized Nehari manifold

$\mathscr{M}=\left\{z \in E \backslash E^{-}:\left\langle I^{\prime(z)}, z\right\rangle=0\right.$ and $\left\langle I^{\prime(z)}, \eta\right\rangle=0$ for all $\left.\eta \in E^{-}\right\}$.

We need to prove that $\mathscr{M} \neq \varnothing$.

For $z \in E \backslash E^{-}$, define $\gamma \in C^{1}\left(\mathbb{R}^{+} \times F, \mathbb{R}\right)$ given by

$$
\gamma_{z}(t, \eta)=I\left(t z^{+}+\eta\right)
$$

It is easy to check the following lemma (see, e.g., Lemma 3.1 in [25]).

Lemma 4. $(t, \eta) \in \mathbb{R}^{+} \times E^{-}$is a critical point of $\gamma_{z}$ if and only if $t z^{+}+\eta \in \mathscr{M}$.

For $e \in E \backslash E^{-}$, set $\widehat{E}(e):=E^{-} \oplus \mathbb{R}^{+} e$, where $\mathbb{R}^{+}=[0,+\infty)$.

Lemma 5. For each $z \in E \backslash E^{-}$, there exists $t_{z} z^{+}+\eta_{z} \in \widehat{E}(z)$, such that

$$
I\left(t_{z} z^{+}+\eta_{z}\right)=\max _{t \geq 0, \eta \in F} I\left(t z^{+}+\eta\right)
$$

Moreover, $t_{z} z^{+}+\eta_{z} \in \mathscr{M}$.

Proof. By Lemma 4, it suffices to prove that the maximum exists. This follows from the next two lemmas. Indeed, using Lemma 7 and Lemma 8, combining with $I(0)=0$, it is easy to check that the maximum point exists.

Remark 6. Even though $f$ and $g$ have satisfied the condition $\left(S_{5}\right)$ in [23], we cannot have the uniqueness of $t_{z} z^{+}+\eta_{z}$. So, we cannot get the result similar to Proposition 2.3 in [23].

Lemma 7. There are $r>0$ and $\rho>0$ such that $\inf _{z \in S_{\rho}^{+}} I(z) \geq r$, where $S_{\rho}^{+}=\left\{z \in E^{+}:\|z\|=\rho\right\}$.
Proof. For a given $\varepsilon>0$, by $\mathrm{H}_{2}, \mathrm{H}_{3}$, and $\mathrm{H}_{4}$, there is a $C(\varepsilon)>0$ such that

$$
|F(u)| \leq \varepsilon|u|^{2}+C(\varepsilon)|u|^{p+1}
$$

By Hardy-Littlewood-Sobolev's inequality, one has

$$
\begin{aligned}
& \int_{\Omega} \int_{\Omega} \frac{F(u(y)) F(u(x))}{|x-y|^{\alpha}} d y d x \\
& \quad \leq C\|F(u)\|_{L^{r}(\Omega)}^{2} \leq C\left(\int_{\Omega}\left(\varepsilon|u|^{2}+C(\varepsilon)|u|^{p+1}\right)^{r} d x\right)^{2 / r} \\
& \quad \leq C_{1} \varepsilon\|z\|^{4}+C_{2} C(\varepsilon)\|z\|^{2(p+1)},
\end{aligned}
$$

where $(2 / r)+(\alpha / N)=2$. Here we use the fact that

$$
2<2 r \leq r(p+1)<\frac{2 N}{N-2 s} .
$$

Similarly, we have

$$
\int_{\Omega} \int_{\Omega} \frac{G(v(y)) G(v(x))}{|x-y|^{\alpha}} d y d x \leq C_{1} \varepsilon\|z\|^{4}+C_{2} C(\varepsilon)\|z\|^{2(p+1)} .
$$

Thus, for $z=\left(u, A^{-t} A^{s} U u\right) \in E^{+}$, it holds that

$$
I(z) \geq \frac{1}{2}\|z\|^{2}-\varepsilon\|z\|^{4}-C_{1}(\varepsilon)\|z\|^{p+1}-C_{2}(\varepsilon)\|z\|^{q+1} .
$$

Hence, we can choose some $r, \rho>0$ such that $I(z) \geq r$ for all $\|z\|=\rho$.

Lemma 8. For every $e \in E^{+} \backslash\{0\}$, there is a $R(e)>0$ such that $I<0$ on $\widehat{E}(e) \backslash B_{R(e)}(0)$.

Proof. If not, there exists $\left\{w_{n}\right\} \subset \widehat{E}(e)$ such that $\left\|w_{n}\right\| \longrightarrow \infty$ and $I\left(w_{n}\right) \geq 0$.

Without loss of generality, we may assume that $e \in E^{+}$, $\|e\|=1$. By doing $\left(w_{n, 1}, w_{n, 2}\right)=w_{n}=t_{n} e+w_{n}^{-}$where $t_{n} \geq 0$. Set

$$
\bar{w}_{n}=\frac{w_{n}}{\left\|w_{n}\right\|}=\frac{t_{n}}{\left\|w_{n}\right\|} e+\frac{w_{n}^{-}}{\left\|w_{n}\right\|}:=s_{n}^{2} e+\eta_{n}^{-} .
$$

Obviously, $1=\left\|\bar{w}_{n}\right\|^{2}=s_{n}^{2}+\left\|\eta_{n}^{-}\right\|^{2}$. Jointly with

$$
\begin{aligned}
0 \leq & \frac{I\left(w_{n}\right)}{\left\|w_{n}\right\|^{2}}=\frac{1}{2}\left(s_{n}^{2}-\left\|\eta_{n}^{-}\right\|^{2}\right) \\
& -\frac{1}{2} \int_{\Omega} \int_{\Omega} \frac{F\left(w_{n, 1}(y)\right) F\left(w_{n, 1}(x)\right)}{|x-y|^{\alpha}\left\|w_{n}\right\|^{2}} d y d x \\
& -\frac{1}{2} \int_{\Omega} \int_{\Omega} \frac{G\left(w_{n, 2}(y)\right) G\left(w_{n, 2}(x)\right)}{|x-y|^{\beta}\left\|w_{n}\right\|^{2}} d y d x \\
\leq & \frac{1}{2}\left(s_{n}^{2}-\left\|\eta_{n}^{-}\right\|^{2}\right),
\end{aligned}
$$


one has $(1 / \sqrt{2}) \leq s_{n} \leq 1$. So, for a subsequence, $s_{n} \longrightarrow s>0$, $\bar{w}_{n} \rightarrow \bar{w}, \bar{w}_{n}(x) \longrightarrow \bar{w}(x)$, a.e., in $\Omega$. Hence, $\bar{w}=s e+\bar{w}^{-}$ $(x) \neq 0$. It means that for

$$
\mathscr{A}:=\{x \in \Omega: \bar{w}(x) \neq 0\},
$$

where $|\mathscr{A}|>0$. Thus, for $x \in \mathscr{A},\left|w_{n}(x)\right| \longrightarrow \infty$.

It follows from $\mathrm{H}_{2}, \mathrm{H}_{3}$, and $\mathrm{H}_{5}$ that there exists $C_{1}, C_{2}>0$ such that

$$
\begin{aligned}
& F(u) \geq C_{1}|u|^{\theta}-C_{2}, \\
& G(v) \geq C_{1}|v|^{\theta}-C_{2},
\end{aligned}
$$

and

$$
\begin{aligned}
& \lim _{u \rightarrow \infty} \frac{F(u)}{u^{2}}=\infty \\
& \lim _{v \rightarrow \infty} \frac{G(v)}{v^{2}}=\infty .
\end{aligned}
$$

Thus, $w_{n}=\left(w_{n, 1}, w_{n, 2}\right)$, it follows from Fatou's lemma that

$$
\begin{aligned}
& \int_{\Omega} \int_{\Omega} \frac{F\left(w_{n, 1}(y)\right) F\left(w_{n, 1}(x)\right)}{|x-y|^{\alpha}\left\|w_{n}\right\|^{2}} d y d x \\
&+\int_{\Omega} \int_{\Omega} \frac{G\left(w_{n, 2}(y)\right) F\left(w_{n, 2}(x)\right)}{|x-y|^{\beta}\left\|w_{n}\right\|^{2}} d y d x \\
& \geq C\left(\int_{\mathscr{A}} C_{1}\left|w_{n}\right|^{\theta} d x-C_{2}|\mathscr{A}|\right) \int_{\mathscr{A}} \frac{F\left(w_{n, 1}\right)+G\left(w_{n, 2}\right)}{\left|w_{n}\right|^{2}} \frac{\left|w_{n}\right|^{2}}{\left\|w_{n}\right\|^{2}} d x \\
& \quad \geq C_{3} \int_{\mathscr{A}} \frac{F\left(w_{n, 1}\right)+G\left(w_{n, 2}\right)}{\left|w_{n}\right|^{2}} \frac{\left|w_{n}\right|^{2}}{\left\|w_{n}\right\|^{2}} d x \longrightarrow \infty,
\end{aligned}
$$

which would conflict with (38). Here, we use the fact that $\Omega$ is bounded.

\section{Lemma 9. $M$ contains all nontrivial critical points.}

Proof. If $z=(u, v) \in E, z \neq 0$, and $I^{\prime}(z)=0$, by $\mathrm{H}_{5}$ and $\mathrm{H}_{6}$, one has

$$
\begin{aligned}
I(z)= & I(z)-\frac{1}{2}\left\langle I^{\prime}(z), z\right\rangle \\
= & \frac{1}{2} \int_{\Omega} \int_{\Omega} \frac{F(u(y))[f(u(x)) u(x)-F(u(x))]}{|x-y|^{\alpha}} d y d x \\
& +\frac{1}{2} \int_{\Omega} \int_{\Omega} \frac{G(v(y))[g(v(x)) v(x)-G(v(x))]}{|x-y|^{\beta}} d y d x \\
\geq & C_{1} \int_{\Omega} F(u) d x \int_{\Omega}[f(u) u-F(u)] d x \\
& +C_{2} \int_{\Omega} G(v) d x \int_{\Omega}[g(v) v-G(v)] d x>0 .
\end{aligned}
$$

For $z \in E^{-} \backslash\{0\}$, it follows that

$$
\begin{aligned}
I(z)= & -\frac{1}{2}\left\|z^{-}\right\|^{2}-\int_{\Omega} \int_{\Omega} \frac{F(u(y)) F(u(x))}{|x-y|^{\alpha}} d y d x \\
& -\int_{\Omega} \int_{\Omega} \frac{G(u(y)) G(u(x))}{|x-y|^{\beta}} d y d x \leq 0 .
\end{aligned}
$$

Define

$$
m:=\inf _{z \in \mathscr{M}} I(z)
$$

We shall prove that $m$ is achieved on $\mathscr{M}$ and the minimizer is a critical point of $I$.

\section{Lemma 10.}

(1) There exists $\rho>0$ such that

$$
m=\inf _{z \in \mathscr{M}} I(z) \geq \inf _{z \in S_{\rho}} I(z)>0,
$$

where $S_{\rho}:=\left\{z \in E^{+}:\|z\|=\rho\right\}$.

(2) $\left\|z^{+}\right\| \geq \max \left\{\left\|z^{-}\right\|, \sqrt{2 m}\right\}$ for every $z \in \mathscr{M}$.

Proof. First, let us prove assertion (1). For every $z \in \mathscr{M}$, with Lemma 7 in hand, we can choose some $\delta>0$, small $\rho>0$ such that

$$
I(z) \geq I\left(\rho \frac{z^{+}}{\left\|z^{+}\right\|}\right) \geq \delta .
$$

Second, $I(z) \leq 1 / 2\left(\left\|z^{+}\right\|^{2}-\left\|z^{-}\right\|^{2}\right)$ for every $z \in \mathscr{M}$, which concludes the proof of assertion (2).

Lemma 11. I is coercive on $\mathscr{M}$.

Proof. If not, there exists a sequence $\left\{z_{n}\right\} \subset \mathscr{M}$ such that $\left\|z_{n}\right\| \longrightarrow \infty$ and $I\left(z_{n}\right) \leq d$ for some $d \in[c, \infty)$. Let $w_{n}=$ $\left(w_{n, 1}, w_{n, 2}\right):=\left(z_{n} /\left\|z_{n}\right\|\right)$. Up to a subsequence, $w_{n} \rightarrow w$ and $w_{n}(x) \longrightarrow w(x)$, a.e., in $\mathbb{R}^{N}$.

If $w=0$, one has $w_{n}^{+} \longrightarrow L^{p}(\Omega) \times L^{q}(\Omega)$ for $1 \leq p<$ $(N-2 s)), 1 \leq q<(2 N /(N-2 t))$. So it holds that

$$
\begin{aligned}
& \lim _{n \rightarrow \infty} \int_{\Omega} \int_{\Omega} \frac{F\left(w_{n, 1}^{+}(y)\right) F\left(w_{n, 1}^{+}(x)\right)}{|x-y|^{\alpha}} d y d x=0, \\
& \lim _{n \rightarrow \infty} \int_{\Omega} \int_{\Omega} \frac{G\left(w_{n, 2}^{+}(y)\right) G\left(w_{n, 2}^{+}(x)\right)}{|x-y|^{\beta}} d y d x=0 .
\end{aligned}
$$

Jointly with Lemma 5, we obtain

$$
d \geq I\left(z_{n}\right) \geq I\left(s w_{n}^{+}\right) \geq \frac{s^{2}}{4}+o_{n}(1)
$$

It is absurd if $s>\sqrt{4 d}$. So $w \neq 0$. 
Similar to the proof of Lemma 7, it holds that

$$
\begin{aligned}
\int_{\Omega} \int_{\Omega} & {\left[\frac{F\left(z_{n, 1}(y)\right) F\left(z_{n, 1}(x)\right)}{|x-y|^{\alpha} z_{n}^{2}}+\frac{G\left(z_{n, 2}(y)\right) F\left(z_{n, 2}(x)\right)}{|x-y|^{\beta} z_{n}^{2}}\right] w_{n}^{2} d y d x } \\
& \longrightarrow{ }^{n \rightarrow \infty} \infty .
\end{aligned}
$$

Therefore,

$$
\begin{aligned}
0 \leq & \frac{I\left(z_{n}\right)}{\left\|z_{n}\right\|^{2}}=\frac{1}{2}\left(\left\|w_{n}^{+}\right\|^{2}-\left\|w_{n}^{-}\right\|^{2}\right) \\
& -\int_{\Omega} \int_{\Omega}\left[\frac{F\left(z_{n, 1}(y)\right) F\left(z_{n, 1}(x)\right)}{|x-y|^{\alpha} z_{n}^{2}}\right. \\
& \left.+\frac{G\left(z_{n, 2}(y)\right) F\left(z_{n, 2}(x)\right)}{|x-y|^{\beta} z_{n}^{2}}\right] w_{n}^{2} d y d x \longrightarrow-\infty,
\end{aligned}
$$

which is a clear contradiction.

Lemma 12. $c=\inf _{z \in M} I(z)$ is achieved.

Proof. The proof is similar to Lemma 3.6 in [33], but for readers' convenience, we review the details of the proof.

Let $\left\{z_{n}\right\} \subset \mathscr{M}$ be a minimizing sequence. In the light of Lemma $10,\left\{z_{n}\right\}$ is bounded in E. Extracting a subsequence if necessary, we have $z_{n} \rightarrow z$ in $E$. By the continuity of the projections, $z_{n}^{+} \rightarrow z^{+}$.

Now let us prove that $z^{+} \neq 0$. If not, by Lemma 5 and the result (2) of Lemma 9, we get

$$
m+o_{n}(1)=I\left(z_{n}\right) \geq I\left(t z_{n}^{+}\right)=\frac{1}{2} t^{2}\left\|z_{n}^{+}\right\|^{2}+o_{n}(1) .
$$

It is impossible if $t$ is large.

By Lemma 5, there exists $t_{z}>0, \eta_{z} \in E^{-}$such that

$$
t_{z} z^{+}+\eta_{z}=\left(\left(t_{z} z^{+}+\eta_{z}\right)_{1},\left(t_{z} z^{+}+\eta_{z}\right)_{2}\right) \in \mathscr{M} .
$$

With Lebesgue's dominated theorem, it follows that

$$
\begin{aligned}
m \leq & I\left(t_{z} z^{+}+\eta_{z}\right)=\frac{1}{2} t_{z}^{2}\left\|z^{+}\right\|^{2}-\frac{1}{2}\left\|\eta_{z}^{-}\right\|^{2} \\
& -\int_{\Omega} \int_{\Omega} \frac{F\left(\left(t_{z} z^{+}+\eta_{z}\right)_{1}(y)\right) F\left(\left(t_{z} z^{+}+\eta_{z}\right)_{1}(x)\right)}{|x-y|^{\alpha}} d y d x \\
& \left.-\int_{\Omega} \int_{\Omega} \frac{G\left(\left(t_{z} z^{+}+\eta_{z}\right)_{2}(y)\right) G\left(\left(t_{z} z^{+}+\eta_{z}\right)_{2}(x)\right)}{|x-y|^{\beta}}\right] d y d x \\
= & \liminf _{n \rightarrow \infty} I\left(t_{z} z_{n}^{+}+\eta_{z}\right) \leq \liminf _{n \rightarrow \infty} I\left(z_{n}\right)=c .
\end{aligned}
$$

Lemma 13. If $z_{0} \in \mathscr{M}$ is such that

$$
m=\inf _{z \in \mathscr{M}} I(z)=I\left(z_{0}\right),
$$

then $I^{\prime}\left(z_{0}\right)=0$.

Proof. Assume by contradiction that $I^{\prime}\left(z_{0}\right) \neq 0$, then $\delta>0$ and $\theta>0$ exist such that

$$
\left\|I^{\prime}\left(z_{0}\right)\right\|_{E^{*}} \geq \theta, \quad \text { for } z \in B_{2 \delta}\left(z_{0}\right) \cap \mathscr{B},
$$

where $\mathscr{B}:=\left\{z \in \widehat{E}\left(z_{0}\right): I(z)<m-\varepsilon\right\}$ (if necessary, we can choose another $z_{0}$ and $\varepsilon$ small enough such that $B_{2 \delta}\left(z_{0}\right) \cap$ $\mathscr{B} \neq \varnothing)$.

Define

$$
T: \widehat{E}\left(z_{0}\right) \longrightarrow \mathbb{R}^{+} \times E^{-},
$$

given by $T\left(t z_{0}^{+}+\eta\right)=(t, \eta)$.

Denote $B_{\delta / 4}:=B_{\delta / 4}\left(z_{0}\right) \cap \mathscr{B}, \tilde{T}:=\left.T\right|_{B_{\delta / 4}}$, and $D:=\tilde{T}\left(B_{\delta / 4}\right)$. Define

$$
\pi:=\tilde{T}^{-1}: D \longrightarrow B_{\delta / 4}
$$

given by $\pi(t, \eta)=t z_{0}^{+}+\eta$.

It is clear that

$$
\max _{(t, \eta) \in \partial D} I(\pi(t, \eta)) \leq m-\varepsilon<m
$$

For

$$
\begin{aligned}
& 0<\varepsilon<\min \left\{\frac{\varepsilon}{2}, \frac{\theta \delta}{8}\right\}, \\
& S:=B_{2 \delta}\left(z_{0}\right) \cap \mathscr{B},
\end{aligned}
$$

it yields a deformation $\Gamma$ (see Lemma 2.3 of [34] or A.4 of [35]) such that

(a) $\Gamma(1, z)=z$ if $z \in I^{-1}([m-2 \varepsilon, m+2 \varepsilon]) \cap S_{2 \delta}$,

(b) $\Gamma\left(1, I_{m+\varepsilon} \cap S\right) \subset I_{m-\varepsilon}$, where $S_{2 \delta}:=\{z: \operatorname{dist}(z, S) \leq$ $2 \delta\}$.

Let us define a $h: D \longrightarrow E$ and for every $w \in E^{-}, \Psi_{0}^{w}$, $\Psi_{1}^{w}: D \longrightarrow \mathbb{R}^{2}$ by

$$
\begin{aligned}
h(t, \eta) & =\Gamma(1, \pi(t, \eta)), \\
\Psi_{0}^{w}(t, \eta) & \left.=\left(\left\langle I^{\prime(\pi(t, \eta))}, \pi(t, \eta)\right\rangle,\left\langle I^{\prime(\pi(t, \eta))}, w\right)\right\rangle\right), \\
\Psi_{1}^{w}(t, \eta) & \left.=\left(\left\langle I^{\prime}(h(t, \eta)), h(t, \eta)\right\rangle,\left\langle I^{\prime}(h(t, \eta)), w\right)\right\rangle\right) .
\end{aligned}
$$

Lemma 5 and the degree theory now yield that

$$
\operatorname{deg}\left(\Psi_{0}^{w}, D, 0\right)=1
$$


Since $(t, \eta) \in \tilde{T}\left(\partial B_{\delta / 4}\right)$, combining with (53), it therefore holds that

$$
I((\pi(t, \eta))) \leq m-\varepsilon<m-2 \varepsilon .
$$

It follows from (a) that

$$
\Psi_{0}^{w}=\Psi_{1}^{w} \text { on } \partial D
$$

Consequently, we obtain

$$
\operatorname{deg}\left(\Psi_{1}^{w}, D, 0\right)=1 .
$$

Then, we know that there exists $\left(t_{0}, \eta_{0}\right) \in D$ such that $h\left(t_{0}, \eta_{0}\right) \in \mathscr{M}$. This implies that

$$
m \leq I\left(h\left(t_{0}, \eta_{0}\right)\right)=I\left(\Gamma\left(1, \pi\left(t_{0}, \eta_{0}\right)\right)\right) .
$$

Noting that $I\left(\pi\left(t_{0}, \eta_{0}\right)\right)<m+\varepsilon$ and also $\pi(D)=B_{\delta / 4}$ $C S$, in view of (b), one has $m \leq I\left(\Gamma\left(1, \pi\left(t_{0}, \eta_{0}\right)\right)\right) \leq m-\varepsilon$, which is a clear contradiction.

Proof of Theorem 14. The existence of $(s, t)$ ground state solution of (1) follows directly from Lemma 12 to Lemma 13.

\section{Data Availability}

Data are available upon request. All data will be such that no personal identifying information will be included.

\section{Conflicts of Interest}

The authors declare that there is no conflict of interest regarding the publication of this paper.

\section{Authors' Contributions}

The authors contributed to each part of this paper equally. The authors read and approved the final manuscript.

\section{Acknowledgments}

This work is supported by the National Natural Sciences Foundation of People's Republic of China under Grants 11901514, 11861072, 11961078, and 11561072.

\section{References}

[1] E. H. Lieb, "Existence and uniqueness of the minimizing solution of Choquard's nonlinear equation," Studies in Applied Mathematics, vol. 57, no. 2, pp. 93-105, 1976.

[2] V. Moroz and J. Van Schaftingen, "Groundstates of nonlinear Choquard equations: existence, qualitative properties and decay asymptotics," Journal of Functional Analysis, vol. 265, no. 2, pp. 153-184, 2013.

[3] R. Penrose, "On gravity's role in quantum state reduction," General Relativity and Gravitation, vol. 28, no. 5, pp. 581600, 1996.

[4] P. L. Lions, "The Choquard equation and related questions," Nonlinear Analysis, vol. 4, no. 6, pp. 1063-1072, 1980.
[5] C. O. Alves, A. B. Nobrega, and M. Yang, "Multi-bump solutions for Choquard equation with deepening potential well," Calculus of Variations and Partial Differential Equations, vol. 55 , no. 3 , p. $48,2016$.

[6] C. O. Alves and M. Yang, "Multiplicity and concentration of solutions for a quasilinear Choquard equation," Journal of Mathematical Physics, vol. 55, no. 6, article 061502, 2014.

[7] V. Ambrosio, "Multiplicity and concentration results for a fractional Choquard equation via penalization method," Potential Analysis, vol. 50, no. 1, pp. 55-82, 2019.

[8] G. D. Li and C. L. Tang, "Existence of ground state solutions for Choquard equation involving the general upper critical Hardy-Littlewood-Sobolev nonlinear term," Communications on Pure \& Applied Analysis, vol. 18, no. 1, pp. 285-300, 2019.

[9] L. Ma and L. Zhao, "Classification of positive solitary solutions of the nonlinear Choquard equation," Archive for Rational Mechanics and Analysis, vol. 195, no. 2, pp. 455-467, 2010.

[10] P. Ma and J. Zhang, "Existence and multiplicity of solutions for fractional Choquard equations," Nonlinear Analysis, vol. 164, pp. 100-117, 2017.

[11] G. P. Menzala, "On regular solutions of a nonlinear equation of Choquard's type," Proceedings of the Royal Society of Edinburgh: Section A Mathematics, vol. 86, no. 3-4, pp. 291-301, 1980.

[12] Z. Shen, F. Gao, and M. Yang, "Ground states for nonlinear fractional Choquard equations with general nonlinearities," Mathematical Methods in the Applied Sciences, vol. 39, pp. 4082-4098, 2016.

[13] S. Bhattarai, "On fractional Schrödinger systems of Choquard type," Journal of Differential Equations, vol. 263, no. 6, pp. 3197-3229, 2017.

[14] J. Giacomoni, T. Mukherjee, and K. Sreenadh, "Doubly nonlocal system with Hardy-Littlewood Sobolev critical nonlinearity," http://arxiv.org/abs/1711.02835v1.

[15] F. Gao, E. D. da Silva, M. Yang, and J. Zhou, "Existence of solutions for critical Choquard equations via the concentration compactness method," Proceedings of the Royal Society of Edinburgh: Section A Mathematics, vol. 150, no. 2, pp. 921954, 2020.

[16] F. Gao and M. Yang, "On nonlocal Choquard equations with Hardy-Littlewood-Sobolev critical exponents," Journal of Mathematical Analysis and Applications, vol. 448, no. 2, pp. 1006-1041, 2017.

[17] F. Gao and M. Yang, "A strongly indefinite Choquard equation with critical exponent due to the Hardy-Littlewood-Sobolev inequality," Communications in Contemporary Mathematics, vol. 20, no. 4, article 1750037, 2018.

[18] Y. Ding, Variational Methods for Strong Infinity Problems, World Scientific Press, 2008.

[19] D. G. de Figueiredo and P. L. Felmer, "On superquadratic elliptic systems," Transactions of the American Mathematical Society, vol. 343, no. 1, pp. 99-116, 1994.

[20] L. Fang and Y. Jianfu, "Nontrivial solutions of Hardy-Hénon type elliptic systems," Acta Mathematica Scientia, vol. 27, no. 4, pp. 673-688, 2007.

[21] F. Zhao and Y. Ding, "On Hamiltonian elliptic systems with periodic or non-periodic potentials," Journal of Differential Equations, vol. 249, no. 12, pp. 2964-2985, 2010.

[22] F. Zhao, L. Zhao, and Y. Ding, "Multiple solutions for a superlinear and periodic elliptic system on $\mathbb{R}^{N}$," Zeitschrift für 
Angewandte Mathematik und Physik, vol. 62, no. 3, pp. 495$511,2011$.

[23] A. Szulkin and T. Weth, "Ground state solutions for some indefinite variational problems," Journal of Functional Analysis, vol. 257, no. 12, pp. 3802-3822, 2009.

[24] A. Szulkin and T. Weth, The Method of Nehari Manifold, Handbook of Nonconvex Analysis and Applications, Boston: International Press, 2010.

[25] G. M. Figueiredo and M. T. O. Pimenta, "Existence of ground state solutions to Dirac equations with vanishing potentials at infinity," Journal of Differential Equations, vol. 262, no. 1, pp. 486-505, 2017.

[26] E. H. Lieb and M. Loss, "Analysis," in Graduate Studies in Mathematics, vol. 14, American Mathematical Society, Providence, Atlanta, GA, 2nd edition, 2001.

[27] T. Bartsch and D. Figueiredo, "Infinitely many solutions of nonlinear elliptic systems, infinitely many solutions of nonlinear elliptic systems," Progress in Nonlinear Differential Equations and their Applications, vol. 35, pp. 51-67, 1999.

[28] M. Reed and B. Simon, Methods of Modern Mathematical Physics, I, Fourier Analysis, Self Adjointness, Academic Press, New York, 1978.

[29] D. E. Edmunds and W. D. Evans, Spectral Theory and Differential Operators, Oxford University Press, New York, 1987.

[30] T. Kato, Perturbation Theory for Linear Operator, SpringerVerlag, New York, Heidelberg, Berlin, Tokyo, 2nd edition, 1984.

[31] K. Chang and M. Guo, Teaching Materials of Functional Analysis, Peking University Press, Chinese, 1990.

[32] H. Triebel, Interpolation Theory, Function Spaces, Differential Operators, Amsterdam, North-Holland, 1978.

[33] W. WANG and Q. LI, "Existence of ground state solutions to Hamiltonian elliptic system with potentials," Acta Mathematica Scientia, vol. 38, no. 6, pp. 1966-1980, 2018.

[34] M. Willem, Minimax Theorems, Progress in Nonlinear Differential Equations and Their Applications, 24, Birkhäuser Boston, Inc., Boston, MA, 1996.

[35] P. H. Rabinowitz, "Minimax methods in critical point theory with application to differential equations," in CBMS Reg. Conf. Ser. Math, vol. 65, American Mathematical Society, Providence, RI, 1986. 